\title{
The Coherent X-ray Sub-micro and Nanoprobe Stations for the CARNAÚBA Beamline at the Sirius-LNLS Storage Ring.
}

Carlos A. Pérez ${ }^{1}$, Hélio C. N. Tolentino ${ }^{1,2, *}$, Márcio M. Soares ${ }^{1}$, Carlos S. B. Dias ${ }^{1}$, Douglas Galante ${ }^{1}$, Verônica de C. Teixeira ${ }^{1}$, Dalton Abdala ${ }^{1}$, Harry Westfahl Jr. .

1. Laboratório Nacional de Luz Síncrotron, LNLS, CNPEM, CP 6192,13085-970 Campinas, Brazil.

2. Université de Grenoble Alpes and CNRS, Institut Néel, BP 166, 38042 Grenoble, France.

* Corresponding author, helio.tolentino@lnls.br

CARNAÚBA (Coherent X-Ray Nanoprobe Beamline) is the tender-to-hard X-ray nanoprobe under construction at the new Brazilian synchrotron light source SIRIUS [1]. The beamline provides two separated experimental stations, one with sub-micrometer resolution and another with nanometer resolution, to cover various analysis techniques, including XRD, XAS, XRF, XEOL, CDI and ptychography with 2D and 3D imaging capabilities exploring the coherent properties of the light. The innovative modified-Delta undulator, with four independent jaws, able to deliver any polarization, is optimized to provide photons with vertical polarization allowing for scattering in the horizontal plane from the source up to the endstations, with unitary polarization factor. The all achromatic CARNAÚBA optics, based on state-of-theart $\mathrm{KB}$ mirrors, covers continuously the energy range from 2.05 to $15 \mathrm{keV}$ with a resolution of $\Delta \mathrm{E} / \mathrm{E}=10^{-}$ ${ }^{4}$, using an in-house developed four-bounce crystal monochromator. An unprecedent coherent flux @ $\mathrm{Si}(111) \mathrm{BW}$ close to $10^{+11}-10^{+12} \mathrm{ph} / \mathrm{s} / 100 \mathrm{~mA}$ will be delivered [2].

The focus is nearly diffraction limited in the whole energy range for both end-stations. At the nanoprobe, the focus is around $120 \mathrm{~nm}$ at $2.05 \mathrm{keV}$ down to $30 \mathrm{~nm}$ above $8 \mathrm{keV}$, with a quite large numerical aperture of $2 \mathrm{NA} \approx 5 \mathrm{mrad}$; at the sub-microprobe, the optics delivers a larger focus of $500 \mathrm{~nm}$ at $2.05 \mathrm{keV}$ down to $100 \mathrm{~nm}$ above $8 \mathrm{keV}$, limited by a reduced numerical aperture of about 2NA $\approx 1.0 \mathrm{mrad}$ (Figure 1).

The nanoprobe station is an all-in-vacuum $\mathrm{KB}$ optics and sample environment with a working distance of about $50 \mathrm{~mm}$ [2]. A cryogenic sample holder and transfer system is under development. An innovative high-dynamic actuation on the vertical and horizontal KB mirrors provides a fast-scanning capability in the 10 to $100 \mathrm{~Hz}$ range for fast fly-scan operation. We estimate to collect full 100x100 ptychographic projections in tens of seconds, taking advantage of the full capability of the in-house developed area detector based on the Medipix 3RX chips.

On the other hand, the sub-microprobe has a much larger working distance, with nearly $450 \mathrm{~mm}$ from the horizontal mirror to the sample stage. The increased working distance comes with a reduced numerical aperture $(1.0 \mathrm{mrad})$ that is, in fact, better suited for diffraction experiments. The sub-microprobe optics employs reduced mirror sizes compared to the nanoprobe, but requires essentially the same level of polishing quality. Both stations require mirrors with RMS slope, height and peak-to-valley errors smaller than $100 \mathrm{nrad}, 1.0 \mathrm{~nm}$ and $3.5 \mathrm{~nm}$, respectively. The UHV environment of the sub-micrometer KB mirrors is separated from the sample stage, whose holder is not in vacuum. In this sense, the station is more flexible with a suitable space around the sample that will allow settling down several kinds of detectors and arrangements, operating in-air or gas flow environments and able to handle up to $5 \mathrm{~kg}$ of sample environment. 
In this contribution, the detailed design and the construction progress of the CARNAÚBA will be reported. Many pieces of instrumentation have been developed at the LNLS and are under commissioning stage.

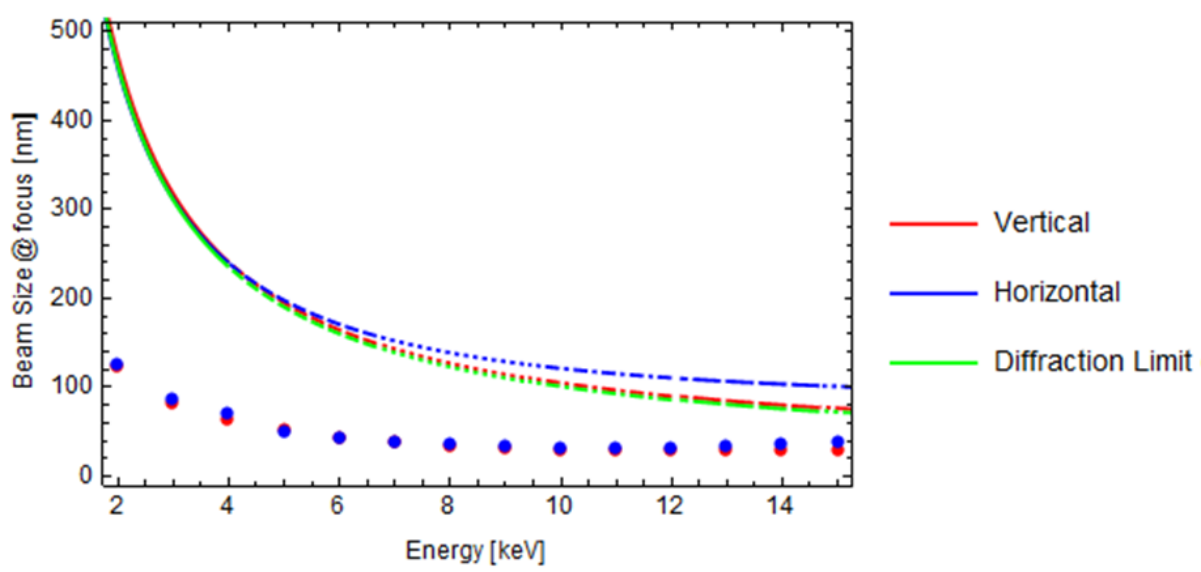

Figure. 1. Energy dependence of the vertical and horizontal focus size for both the sub-microprobe and nanoprobe stations. Lines are for the microprobe; dots are for the nanoprobe. The diffraction limited focus variation is shown for the sub-microprobe.

Among the most important, the modified Delta undulator, a four-bounce crystal monochromator scattering in the horizontal plane, a secondary source aperture working under the pink beam and based on a cryogenically cooled silicon crystal, and a prototype sample manipulation system developed for the sub-microprobe. We expect to have soon in the future a coherent X-ray beamline with two end-stations able to explore a large variety of scientific areas and tackle fundamental questions in science [3].

\section{References:}

[1] webpage of the Sirius project - http://lnls.cnpem.br/sirius/?la=en.

[2] Tolentino et al, Journal of Physics: Conf. Series 849 (2017) 012057.

[3] Technical support from all LNLS groups are acknowledge, in special the assistance and calculations done by Sérgio A. L. Luiz and Bernd C. Meyer from the Optics Group. 